\title{
Microlenses with annular amplitude and phase masks
}

\section{Paeder \\ vincent.paeder@unine.ch \\ T. Scharf \\ P. Ruffieux \\ H.-P. Herzig \\ R. Voelkel \\ K. J. Weible}

\author{
University of Neuchâtel, Institute of Microtechnology, Rue A.-L. Breguet 2, 2000 Neuchâtel, Switzer- \\ land \\ University of Neuchâtel, Institute of Microtechnology, Rue A.-L. Breguet 2, 2000 Neuchâtel, Switzer- \\ land \\ University of Neuchâtel, Institute of Microtechnology, Rue A.-L. Breguet 2, 2000 Neuchâtel, Switzer- \\ land \\ University of Neuchâtel, Institute of Microtechnology, Rue A.-L. Breguet 2, 2000 Neuchâtel, Switzer- \\ land \\ SUSS MicroOptics, Rue Jaquet-Droz 7, 2007 Neuchâtel, Switzerland
}

SUSS MicroOptics, Rue Jaquet-Droz 7, 2007 Neuchâtel, Switzerland

We present theoretical and experimental investigations of microlenses with both amplitude and phase masks. The light field in the focal region has been measured with a high resolution Mach-Zehnder interferometer with z-scan. The experimental results show good agreement with simulation. We show that we can obtain effects as diverse as squeezing or expansion in longitudinal and lateral directions, focal point splitting and focal shift. These effects may be of interest for applications such as Shack-Hartmann wavefront sensing, integral imaging, optical tweezers and confocal microscopy. [D0I: 10.2971/je0s.2007.07005]

Keywords: Microlenses, pupil filters, focal point shaping

\section{Introduction}

With standard lenses, the concept of amplitude and phase masks, also known as Toraldo filters, is well known to provide superresolution effects in lateral or/and longitudinal directions, depending on the number of annular zones. Simple algorithms, based on Fraunhofer diffraction integral or Debye approximation, are used to determine the exact shape of the 3D point spread function. On the other hand, the influence of diffraction at the microlens edges is no longer negligible, which require treatments in the frame of Fresnel diffraction or Rayleigh-Sommerfeld diffraction. Moreover, the fabrication techniques suitable for the production of large scale arrays at acceptable costs impose a limit in the number of zones, due to resolution and accuracy.

In our work, we investigate the effect of two and three zone masks of amplitude and phase type. The results show similar behaviours as with macrolenses, that is, squeezing or expansion of the focal point in lateral and transversal direction, and, for phase mask, appreciable focal shift. However, effects such as focal point repelling are specific to such small size structures.

These effects may be used in applications such as optical tweezers [5], superresolving filters, [1,2], confocal microscopy $[3,4]$, or, for long focal length lenses, Shack-Hartmann wavefront sensing, integral imaging [6] and fluerescence detection [7].
Here, we present measurements on microlenses covered with rings of various sizes realised with a lift-off technique in chromium. A high resolution Mach-Zehnder interferometer with $\mathrm{z}$ scan allowed to measure the light field in 3D as well as the quality of the lenses. Additional simulations have been carried out using scalar diffraction theory with different degrees of approximation and coherent beam propagation. It was found both theoretically and experimentally that the field in the focal region of lenses with an aperture can suffer from a squeezing or expansion in axial direction, translation, as well as important side lobe enhancement.

The article is organised as follows. In section 2, we recall the theoretical background of scalar diffraction and extract analytical formulas for the field along the lens axis and in the focal plane. Section 3 is devoted to the measurement procedure. Section 4 presents the sample under investigation, while sections 5 and 6 detail the measurement results. A short discussion is provided in section 7 .

\section{THEOR Y}

Macrolenses with ring apertures, known as Toraldo filters, have already been studied extensively, especially for their superresolving effect. Various papers [8]- [12] detail methods derived from the Fraunhofer diffraction integral. 
Microlenses, down to a certain size, can be treated similarly, by applying the same principles to the first RayleighSommerfeld diffraction integral with various degrees of approximation. In the frame of the thin element approximation (TEA), the method, while providing analytical formulas for some important field features, can give a good impression of the properties of a given filter. Obviously, the resulting ease of implementation comes at the cost of accuracy, particularly for phase masks and smaller filter structures.

In this section, we derive formulas for extracting basic properties such as the intensity along the lens axis and in the focal plane.

Within the frame of Kirchhoff approximation, that is, the field on the lens surface is assumed to be equal to the incident field, the first Rayleigh-Sommerfeld integral stands as:

$$
U(r, \theta, z)=\frac{1}{2 \pi} \int_{0}^{\infty} d r_{0} r_{0} \int_{0}^{2 \pi} d \theta_{0} U_{i n c} \frac{\partial G_{1}}{\partial \hat{n}}
$$

where $\hat{n}$ is the vector normal to the lens surface, $G_{1}$ is the Green's tensor defined as:

$$
G_{1}\left(r, r_{0}, \theta, \theta_{0}, z, z_{0}\right)=\frac{e^{i k \rho}}{\rho}
$$

and $\rho=\sqrt{r^{2}+r_{0}^{2}+2 r r_{0} \cos \left(\theta-\theta_{0}\right)+\left(z-z_{0}\right)^{2}}$.

In the TEA, the lens is approximated by a flat surface with a complex transmission function:

$$
P(r, \theta)=T(r, \theta) \exp \left(i k(n-1) \sqrt{R_{c}^{2}-r^{2}}\right)
$$

with $R_{c}$ being the radius of curvature of the lens, $n$ its refractive index and $T$ a complex valued function which accounts for the influence of the filter.

In the cases, which we are interested in, the focal point lies sufficiently far away for the Fresnel approximation (parabolic wavefronts) to be valid. The pupil function $P$ can be rewritten in the commonly known form:

$$
P(r, \theta)=T(r, \theta) \exp \left(-i k \frac{r^{2}}{2 f}\right)
$$

with $f=R_{c} /(n-1)$.

Considering a lens of radius $R$ surrounded by an opaque mask assumed to be rotationally symmetric, and using the following identity:

$$
J_{0}(z)=\frac{1}{2 \pi} \int_{0}^{2 \pi} e^{i k z \cos \theta} d \theta
$$

the diffraction integral becomes:

$$
\begin{aligned}
U(r, f+\delta)=A(r, f+\delta) \int_{0}^{R} & r_{0} J_{0}\left(\frac{k r_{0} r}{f+\delta}\right) \\
& T\left(r_{0}\right) \exp \left(-j \frac{k \delta r_{0}^{2}}{2 f(f+\delta)}\right) \mathrm{d} r_{0}
\end{aligned}
$$

with

$$
A(r, z)=-\frac{2 \pi j \exp (j k z) \exp \left(j \frac{k r^{2}}{2 z}\right)}{\lambda z}
$$

Analytical expressions for the field along the lens axis and in the geometrical focus plane can be extracted. For a single zone of uniform complex transmission $C$ extending from $r_{a}$ to $r_{b}$, we obtain:

$$
\begin{aligned}
U_{C}(0, f+\delta)=2 j C \frac{f(f+\delta)}{k \delta} & {\left[\exp \left(-i k \frac{\delta r_{b}^{2}}{2 f(f+\delta)}\right)\right.} \\
& \left.-\exp \left(-i k \frac{\delta r_{a}^{2}}{2 f(f+\delta)}\right)\right]
\end{aligned}
$$

At some distance $\delta \neq 0$ from the geometrical focus, the integral (6) cannot be evaluated analytically for $r>0$. However, in the cases when the peak irradiance is situated at the geometrical focus, one can gain useful informations from:

$$
U_{C}(r, f)=A(r, f) C \frac{f}{k r}\left[J_{1}\left(\frac{k r_{b} r}{f}\right)-J_{1}\left(\frac{k r_{a} r}{f}\right)\right]
$$

which gives the contribution from the zone $C$ to the field in the focal plane.

In the following, the integration of Eq. (1) over the lens surface is labelled as $R S$, Eq. (1) in the frame of TEA as $R S-T E A$, and the Fresnel approximation Eq. (6) as Fresnel. The field outside the pupil region is assumed to be zero, restricting the integration to the lens surface.

Further computations made with a commercial software (FRED) based on a coherent beam propagation method [13] are provided for the sake of comparison. They are labeled as Beam in the following discussion. For clarity, the methods are summarised in Table 1.

\begin{tabular}{|c|c|}
\hline$R S$ & Eq. (1) computed over the exact lens profile \\
$R S-T E A$ & Eq. (1) with the thin element approximation \\
Fresnel & Eq. (8) summed over the different zones \\
Beam & Coherent beam propagation method \\
\hline
\end{tabular}

TABLE 1 Summary of the compared simulation methods with their abbreviations.

\section{EXPER I M E T A L SET UP}

The interferometer used in the experiment is described in [14]. The source is a $10 \mathrm{~mW}, 632.8 \mathrm{~nm}$ He-Ne laser. The light scattered by the sample is collected by a $50 \mathrm{x}(\mathrm{NA}=0.85) \mathrm{mi}-$ croscope objective and collimated with a $250 \mathrm{~mm}$ lens. The image is finally recorded by a SONY XC-77 CE 8 bit CCD sensor with $11.5 \mu \mathrm{m}$ pixel size and a resolution of 756x581 pixels. From the microscope resolution, the integration volume is $0.88 \times 0.75^{2} \mu m^{3}$.

The lens quality assessment is performed using an additional 20x $(\mathrm{NA}=0.4)$ objective placed under the sample. 


\section{S A M P LE}

The sample consists of an array of 65x65 fused silica lenses with the characteristics listed in Table 2. They are fabricated by a standard thermal reflow technique and transfered into the substrate by reactive ion etching (RIE) [15]. A $100 \mathrm{~nm}$ thick chromium layer is deposited on top. An annular amplitude mask is created either with a lift-off technique or by wet etching. The chromium structures are then transfered into the fused silica via a further RIE step. Finally, the chromium layer is stripped off, uncovering the resulting phase rings. The same sample has been used for both amplitude and phase masks measurements.

\begin{tabular}{|c|c|}
\hline \multicolumn{2}{|c|}{ General characteristics } \\
\hline Diameter & $80 \mu \mathrm{m}$ \\
\hline Height & $8 \mu \mathrm{m}$ \\
\hline Focal length & $200 \mu \mathrm{m}$ \\
\hline Numerical aperture & 0.20 \\
\hline \multicolumn{2}{|c|}{ Amplitude masks } \\
\hline Average Strehl ratio & $90.53 \%$ \\
\hline Minimum Strehl ratio & $86.64 \%$ \\
\hline \hline \multicolumn{2}{|c|}{ Phase masks } \\
\hline Average Strehl ratio & $90.05 \%$ \\
\hline Minimum Strehl ratio & $87.80 \%$ \\
\hline
\end{tabular}

TABLE 2 General characteristics and quality of measured lenses. The Strehl ratio is measured on diffraction limited lenses after deposition and transfer of the masks.

The quality of 6 diffraction limited lenses, placed across the array diagonal, has been measured. The quality measurements have been performed after the chromium mask deposition and after the phase mask transfer, to assess of the influence of the processes on the lens quality. The Strehl ratio can be seen in Table 2. Though not meeting production standards, the corresponding aberrations are negligible for our purpose.

In the next subsections, we present measurement and simulation results for two zone masks. The rings have a varying inner radius $r_{a}$ and an outer radius larger than or equal to the radius of the lens (see Figure 1). The impact of the diffraction at the outer edge of the ring is negliglible, as it is placed over a strongly refracting area. Therefore, the outer radius $r_{b}$ is taken at most as the lens radius. Results for three zone masks are not presented here. The observed effects are weaker than for two zone structures, and, in the limits imposed by the measurement setup, provide no additional improvements. In reference to Toraldo filters, we may however suppose an influence on the higher order sidelobes.

Furthermore, the quantitative analysis is limited to the peak irradiance position and the peak irradiance full width at half maximum (FWHM) in the longitudinal direction. Its width in the transverse plane is addressed only qualitatively for resolution and interest purposes, the numerical aperture of the lenses being too low for a squeezing to be relevant for superresolution applications.

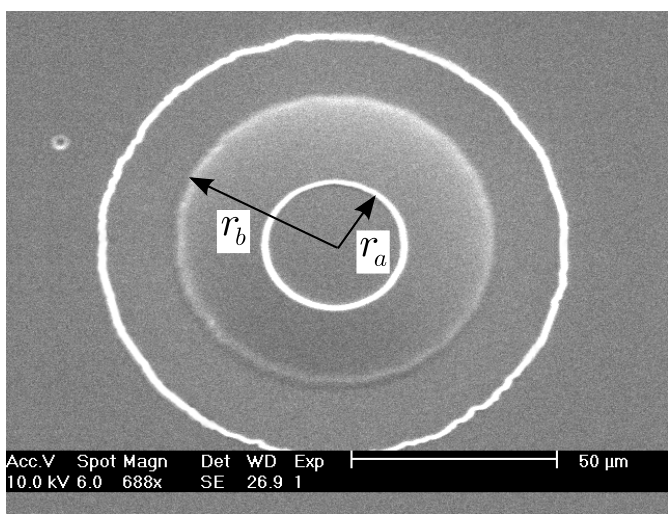

FIG. 1 SEM micrograph of a lens. The ring width is taken as $r_{b}-r_{a}$, while the disc radius as $r_{a}$

\section{A M P LITUDE MASKS}

The chromium layer, is thick enough to be considered as optically opaque. It is therefore acting as an amplitude mask. The role of such a mask is essentially similar to a series of imbricate pinholes. The resulting focal length arises from a combination of refractive effect due to the uncovered portion of the lens and diffractive effects caused by the mask.

A graphical comparison (Figure 2) reveals that the main tendancy is an elongation of the focal spot, doubled with a squeezing in the transverse direction in the case of disc-shape masks. These observations are in concordance with previous results [16].

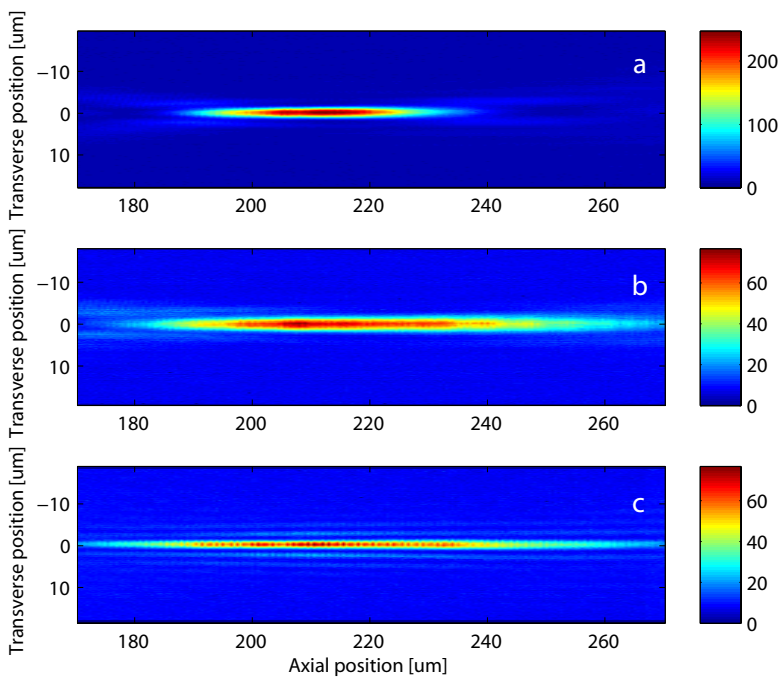

FIG. 2 Comparison of the field intensity in the focal region for a) a simple lens, b) a lens with a $15 \mu \mathrm{m}$ wide chromium ring and c) a lens with a chromium disc of $15 \mu \mathrm{m}$ radius.

\subsection{Peak irradiance position}

As we stated above, the refractive focusing effect of the lens competes with the diffraction at the mask. We expect the peak irradiance position to stay constant until the latter effect overrides the former. For the same focal length, a pinhole with a 
radius of $20 \mu m$ is necessary, which implies that a shift should appear for smaller radii.

For rings, the covered area has a stronger curvature, while the free surface becomes flatter as the ring inner radius is reduced. Therefore, the observed behaviour conforms to the above description well, as seen on Figure 3. A good agreement between experiment and theory can be seen. For small apertures, the pinhole effect taking over the lens is clearly noticeable.

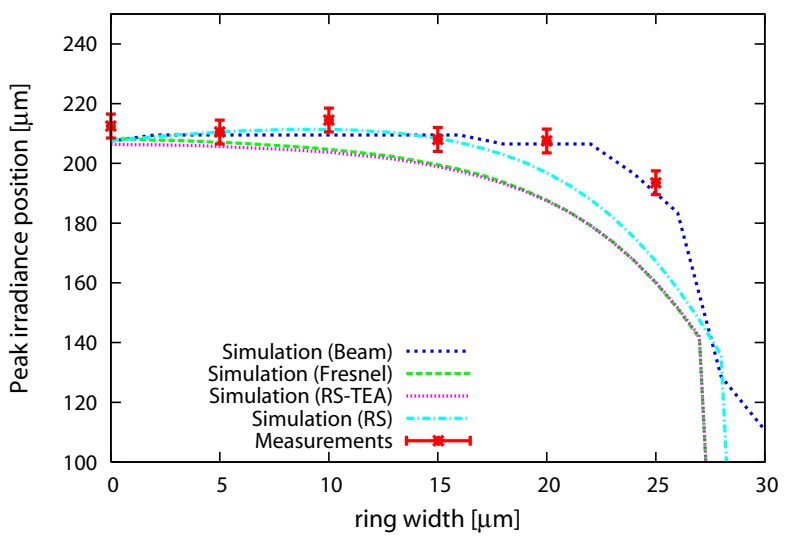

FIG. 3 Position of the peak irradiance as a function of the chromium ring width.

The influence of discs is slightly different than what we would expect from Babinet's principle for two main reasons:

1. the discs cover the less curved area of the lens, leaving a more strongly refracting zone transmissive

2. the opaque zone is, on average, placed further away from the base of the lens than in the case of rings. For that reason, the TEA may be less appropriate.

A comparison between values extracted from measurements and simulations is presented in Figure 4.

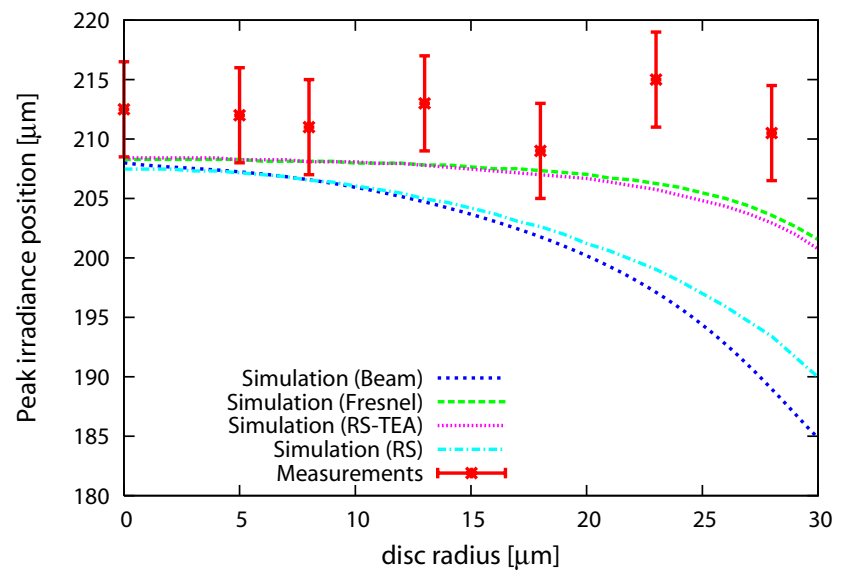

FIG. 4 Position of the peak irradiance as a function of the chromium disc radius. A systematic error arises from the manual positioning of the axial origin.
The TEA reflects well the behaviour of the rings. The beam propagation method is less accurate for reasons which may arise from the density of beams.

\subsection{Peak width along axis}

As one can observe in Figure 2, the focal point becomes noticeably elongated. Pinhole-type and disc-type masks exhibit a similar tendency. However, a stronger effect is observed with pinholes. For rings, we suppose that the diffraction at the mask is not as strongly counterbalanced by the free refractive zone as for discs. A comparison of the full width at half maximum for different disc and ring sizes is presented in Figure 5.
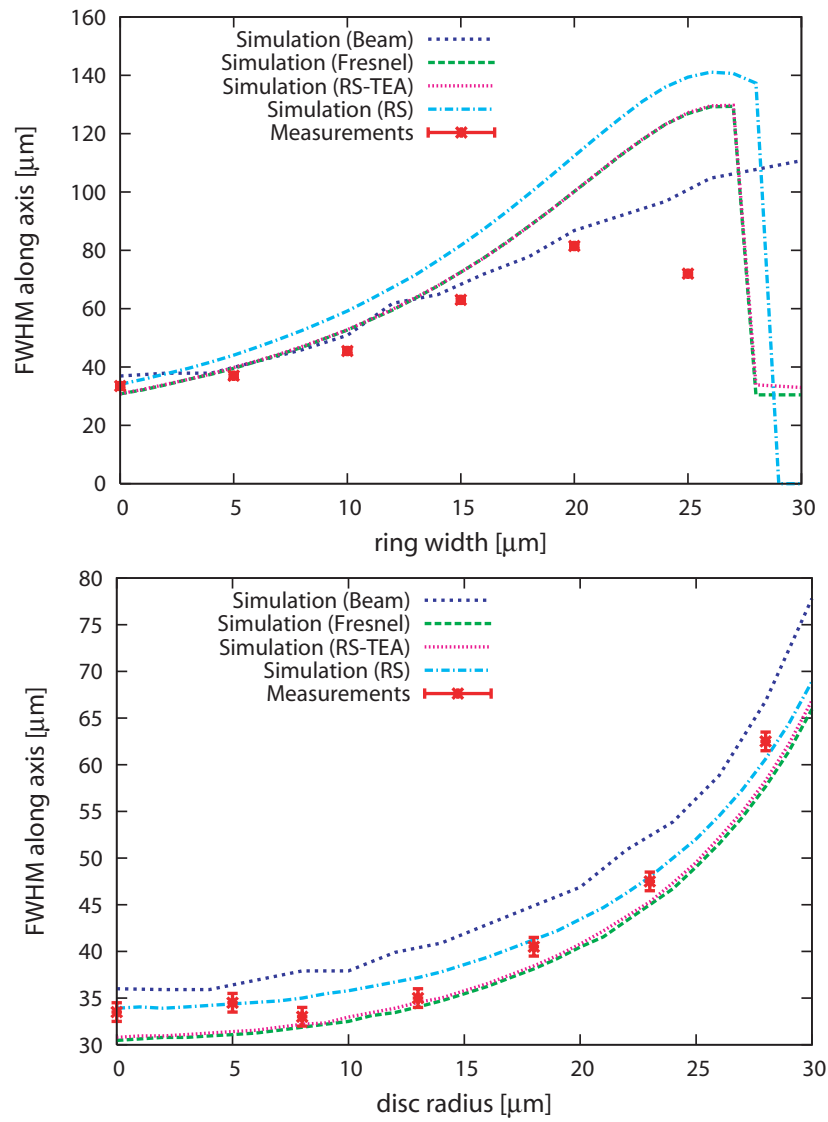

FIC. 5 Peak irradiance FWHM for various chromium ring widths/disc radii.

\section{PHASE MASKS}

The fundamental difference between amplitude and phase masks is the absence of absorption in the latter ones. As a result, their behaviour is better explained as a superposition of two effects. The field refracted by the lens is modulated by the diffraction at the mask. This modulation is observable by the emergence of higher intensity sidelobes along axis (Figure 6), as well as a peak irradiance shift. This shift is induced by the apparition of rays which are out of phase in the focal plane, splitting the maximum to one peak on each side of the focal point.

The impact in the transverse direction is not noticeable as far 
as only the peak irradiance is concerned. Sidelobe enhancement may appear, but this point has not been studied here.

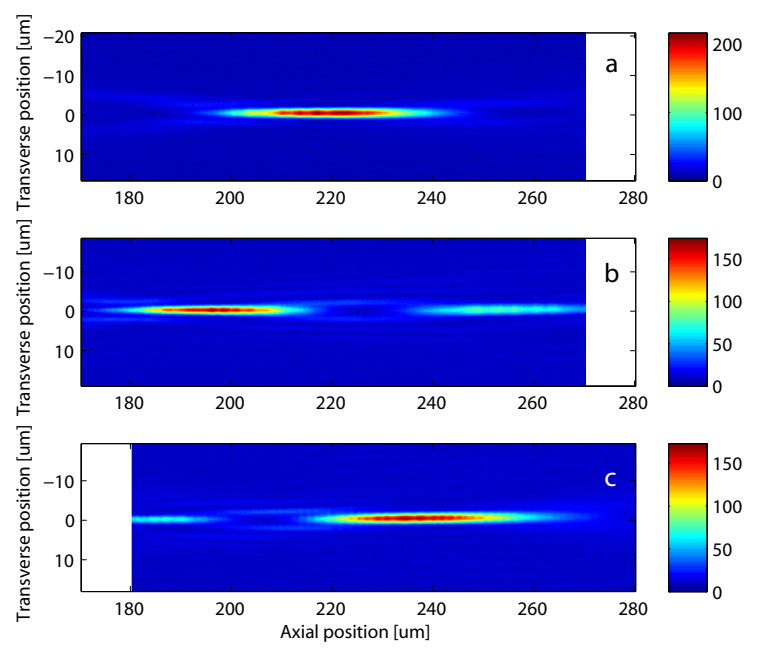

FIG. 6 Comparison of the field intensity in the focal region for a) a simple lens, b) a lens with a $15 \mu \mathrm{m}$ wide phase ring and c) a lens with a phase disc of $15 \mu \mathrm{m}$ radius. The appearance of strong axial sidelobes can be noticed, alongside with a large peak irradiance shift.

\subsection{Peak irradiance position}

As stated above, the peak irradiance position is strongly modified by the influence of the phase structures. This behaviour is observed in Figure 7.
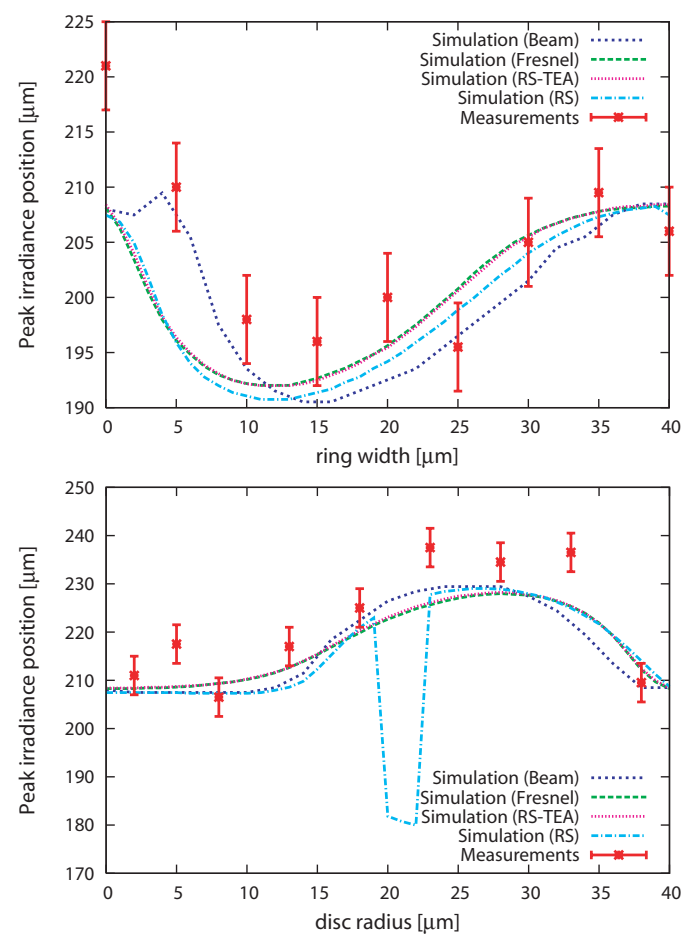

FIG. 7 Peak irradiance position as a function of the ring width/disc radius of the phase filter.

Some divergences with theory are noticeable. They suggest that the control of every aspect of the phase mask-edges, sym- metry and thickness constancy - is critical. In the disc-type case, the RS method shows a switching of the maximum intensity from one peak to the other, indicating that the two peaks intensities are very close to each other.

With the lens characteristics considered here, the position can be varied over a range of almost $30 \%$. Considering that the phase shift of the masks was not optimal, an even more important displacement is reachable.

The presence of a strong axial sidelobe limits the usability for imaging purposes. However, the presence of two peaks, which can be tailored to similar intensities, may be of interest for trapping applications.

\subsection{Peak width along axis}

The shape of the focal spot is largely determined by the strength of the diffraction effects at the mask in comparison to the refraction at the curved surface. When a significant part of the incident field is diffracted away from the focal spot into higher orders, the spot intensity stays high over a wider axial range, as some interfering waves are much weaker. When the mask becomes wider, the importance of diffraction tends to diminish. A superposition of two peaks, emerging from the two imbricate refracting elements, appear.

The described behaviour can be observed at first as a focal spot elongation doubled with a slightly modulated intensity. When the area of the mask becomes wider, the focal spot finally splits into two peaks with uneven intensities.
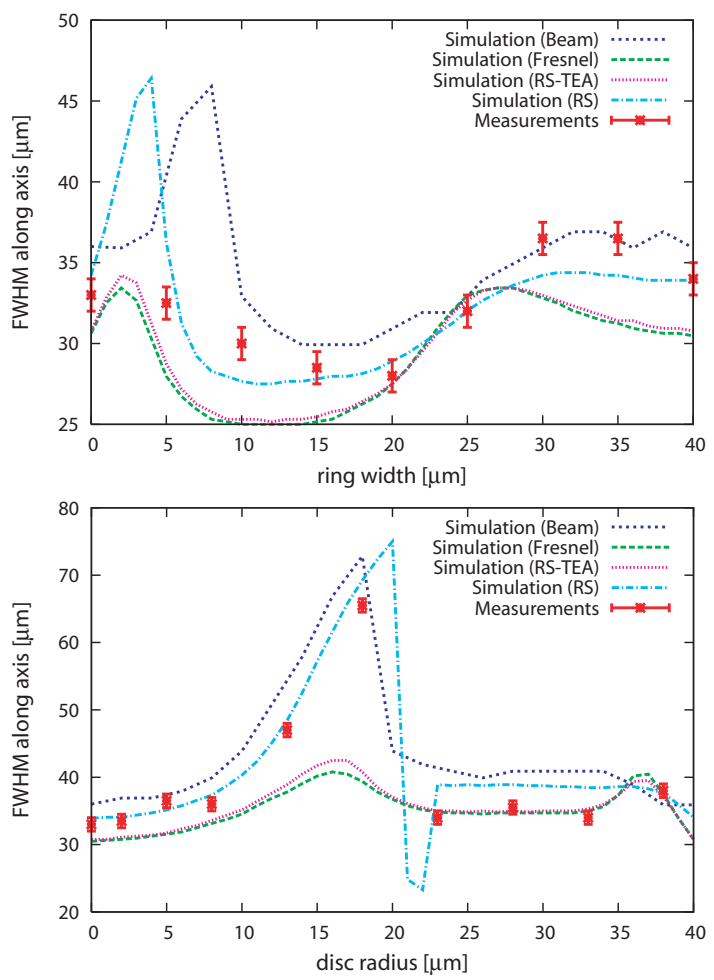

FIG. 8 Peak irradiance width as a function of the ring width/disc radius of the phase filter. 
A summary of the measured and simulated peak irradiance widths is presented in Figure 8.

For discs, the RS method shows superior agreement with measurements. Although the dominant effect is expansion, a squeezing of the order of $10 \%$ is noticeable for several mask sizes. For rings, the size range over which the spot expansion occurs is, according to simulation, small, while the expansion is large. Therefore, we believe that both accuracy of the mask and refinement of the size panel have not been enough to observe it.

\section{DISCUSSION AND CONCLUSION}

In this article, we presented a realisation of microlenses covered with amplitude or phase annular filters. The comparison with different scalar simulation techniques has been performed.

Amplitude masks provide an elongation of the focal point with an additional lateral squeezing for disc-type structures. The measurements are in good agreement with approximations such as thin element approach. This is due to both the opaque nature and the limited thickness $(100 \mathrm{~nm})$ of the masks, which lend themselves well to this type of approximation. Such masks could be of interest in integral imaging, for which a long depth of field is required.

Phase masks present more complex effects due to their completely transmissive nature. They intervene as focal point splitting, peak irradiance shift, expansion or squeezing. The sample under investigation, though not optimised for its influence to be maximised, showed peak irradiance shifts of $\pm 15 \%$ around the original peak irradiance, expansion up to $240 \%$ and squeezing of the order of $10 \%$, depending on the parameters of the annular filter. Obviously, such important effects can be controlled only at the cost of an increased accuracy in production and simulation methods. As we have shown, simple design methodologies based on TEA fail. The masks, only 10 times thinner than the lens itself, cannot be considered to be infinitely thin anymore. Consequently, the RayleighSommerfeld integral with an accurate integration over the lens surface is the only scalar method that stays in agreement with the observations, remaining competitive with the commercial beam propagation software that we employed. This method must be considered with care as to overcome common singularity problems encountered using Eq. (2), which must be dealt with numerically.

As such, the effects obtained with phase masks may attract interest in optical tweezers. A transposition to high numerical aperture lenses is feasible by taking polarisation effects and reflection at the interfaces into account, which implies that scalar methods become invalid. An extension to lenses with very long focal length, such as used for Shack-Hartmann wavefront sensing, may increase the sensitivity of such detectors. Simulation-wise, it is obvious that TEA will fail, as the filters become comparable in thickness to the lens. It must nevertheless be determined, to which extent the physical effects presented here can be transposed to a very different regime.

\section{References}

[1] S. F. Pereira, and A. S. van de Nes, "Superresolution by means of polarisation, phase and amplitude pupil masks" Opt. Commun. 234, 119-124 (2004).

[2] B. Davis, W. Karl, A. Swan, M. Unlu, and B. Goldberg, “Capabilities and limitations of pupil plane filters for superresolution and image enhancement" Opt. Express 12, 4150-4156 (2004).

[3] M. A. A. Neil, R. Juskaitis, T. Wilson, Z. J. Laczik, and V. Sarafis, “Optimized pupil-plane filters for confocal microscope point-spread function engineering" Opt. Lett. 25, 245-247 (2000).

[4] M. Yun, M. Wang, and L. Liu, "Super-resolution with annular binary phase filter in $4 \mathrm{Pi}$ confocal system" J. Opt. A: Pure Appl. 0pt. 7, 640-644, (2005).

[5] C. H. Sow, A. A. Bettiol, Y. Y. G. Lee, F. G. Cheong, C. T. Lim, and F. Watt, "Multiple spot optical tweezers created with microlens arrays fabricated by proton beam writing" Appl. Phys. B 78, 705-709 (2004).

[6] M. Martinez-Corral, B. Javidi, R. Martinez-Cuenca, and G. Saavedra, "Integral imaging with improved depth of field by use of amplitude modulated microlens arrays" Appl. 0pt. 43, 5806-5813 (2004).

[7] J.-C. Roulet, R. Voelkel, H.-P. Herzig E. Verpoorte, N. F. de Rooij, R. Dändliker, "Microlens systems for fluorescence detection in chemical microsystems" Optical Engineering 40, 814-821 (2001).

[8] V. F. Canales, J. E. Oti, and M. P. Cagigal, "Three-dimensional control of the focal light intensity distribution by analytically designed phase masks" Opt. Commun. 247, 11-18 (2005).

[9] M. Martinez-Corral, M. Caballero, E. H. K. Stelzer, and J. Swoger, "Tailoring the axial shape of the point spread function using the Toraldo concept" Opt. Express 10, 98-102 (2002).

[10] D. M. de Juana, J. E. Oti, V. F. Canales, and M. P. Cagigal, “Design of superresolving continuous phase filters" Opt. Lett. 28, 607-609 (2003).

[11] D. M. de Juana, V. F. Canales, P. J. Valle, and M. P. Cagigal, "Focusing properties of annular binary phase filters" Opt. Commun. 229, 71-77 (2004).

[12] S. Ledesma, J. Campos, J. C. Escalera, and M. J. Yzuel, "Symmetry properties with pupil phase-filters" Opt. Express 12, 2548-2559 (2004).

[13] J. Arnaud, "Representation of Gaussian beams by complex rays" Appl. Opt. 24, 538- (1985).

[14] H. Sickinger, J. Schwider, and B. Manzke, "Fiber based MachZehnder interferometer for measuring wave abberations of microlenses" Optik 110, 239-243 (1999).

[15] P. Nussbaum, R. Voelkel, H.-P. Herzig, M. Eisner, and S. Haselbeck, "Design, fabrication and testing of microlens arrays for sensors and microsystems" Pure Appl. Opt. 6, 617-636 (1997).

[16] T. Scharf, P. Ruffieux, W. Noell, R. Voelkel, and C. Rockstuhl, “Light fields generated by microlenses with amplitude stops" in Proceedings of 11 th Microoptics Conference (JSAP, Tokyo, 2005), pp. H-64. 